\title{
Hacia una nueva hegemonía: reflexiones sobre los desafíos para la articulación de las luchas democráticas ${ }^{*}$
}

\author{
María Magdalena Doyle** \\ Valeria Meirovich**
}

Recibido: 2 de mayo de 2013 - Aprobado: 7 de junio de 2013

\begin{abstract}
Resumen
El artículo aborda la propuesta teórica de Chantal Mouffe en relación con la posibilidad de construcción de una democracia radical a partir de la articulación de las luchas por la realización de derechos particulares que llevan a cabo los distintos movimientos sociales.

El análisis que aquí se propone pretende retomar y debatir algunas concepciones asumidas por la autora, al tiempo que incorporar nuevos elementos conceptuales que permitan complejizar la mirada en torno a las posibilidades de hacer efectiva la articulación de luchas por una democracia plena, situando dicha reflexión en el proceso de emergencia de movimientos sociales que en las últimas décadas se ha dado en América Latina y el Caribe.

Palabras clave: Chantal Mouffe, democracia, movimientos sociales, identificación, articulación, condiciones objetivas y subjetivas.
\end{abstract}

\footnotetext{
El artículo se enmarca en las indagaciones teóricas que las autoras realizan como parte de sus investigaciones doctorales, así como también en el Programa de Estudios sobre Comunicación y Ciudadanía del Centro de Estudios Avanzados de la UNC, del cual forman parte.

* Investigadora del Centro de Estudios Avanzados de la Universidad Nacional de Córdoba. Integra el Programa de Estudios sobre Comunicación y Ciudadanía. Becaria, desde el año 2010, del Consejo Nacional de Investigaciones Científica y Técnicas (CONICET) de Argentina. Investiga en torno a los movimientos indígenas en el actual territorio argentino, con énfasis en las luchas de estos movimientos en relación con el derecho a la comunicación. DNI 30.209.221. Correo electrónico: magdalenadoyle@gmail.com

... Docente/Investigadora del Centro de Estudios Avanzados de la Universidad Nacional de Córdoba. Integra el Programa de Estudios sobre Comunicación y Ciudadanía. Becaria, desde el año 2011, del Consejo Nacional de Investigaciones Científica y Técnicas (CONICET) de Argentina. Trabaja en investigación en torno a los movimientos campesinos en Argentina, las prácticas comunicativas y el ejercicio de la ciudadanía. DNI 28.091.317. Correo electrónico: valemeirovich@gmail.com.
} 


\title{
Towards a New Hegemony: Reflections on the Challenges for the Articulation of Democratic Struggles
}

\begin{abstract}
The article addresses the theoretical proposal of Chantal Moufee in relation to the possibility to construct a radical democracy from the articulation of struggles for the performance of individual rights carried out by several social movements.

The analysis proposed is intended to retake and debate on some conceptions assumed by the author, and to incorporate new conceptual elements that allow making complex the idea people have about the possibilities to make articulation of struggles for an entire democracy effective, by placing such a reflection in the emergence process of social movements executed during the last decades in Latin America and the Caribbean.

Key words: Chantal Mouffe; democracy; social movements; identification; articulation; objective and subjective conditions.
\end{abstract}




\section{Introducción}

En las últimas décadas, América Latina y el Caribe han vivido la emergencia de movimientos sociales que cuentan como característica común y central el haber cuestionado y confrontado el desarrollo del capitalismo en su actual fase neoliberal y sus formas de dominación, dando lugar a transformaciones sociales y políticas vinculadas a la obtención de un conjunto de derechos en consonancia con los principios democráticos de libertad e igualdad. Este proceso ha resultado, por demás, significativo en muchos de los países de esta región, revitalizando y profundizando el debate académico que, desde las ciencias sociales, ha configurado a los movimientos sociales como su objeto de estudio.

En este marco, resulta relevante abordar la propuesta desarrollada por Chantal Mouffe en torno a la posibilidad de construcción de una democracia radical a partir de la articulación de las luchas por derechos particulares que llevan a cabo los distintos movimientos sociales.

\section{Metodología}

En ese sentido, el trabajo aquí presentado corresponde a una indagación estrictamente teórica. La propuesta es reflexionar, mediante el análisis de un conjunto de textos de la intelectual politóloga Chantal Mouffe, sobre la producción de dicha autora referida a las posibilidades de construcción de una democracia radical. Conviene aquí aclarar que parte de sus reflexiones han sido elaboradas junto al teórico político Ernesto Laclau, por lo que incorporaremos al análisis Hegemonía y estrategia socialista. Hacia una radicalización de la democracia (2004), libro de su coautoría.

Asimismo, presentamos a lo largo del texto algunas críticas que, esperamos, permitirán enriquecer la comprensión de los procesos de articulación de los movimientos sociales de la región (caribeña y latinoamericana) en la lucha que estos emprenden por la consolidación del orden democrático.

En la medida que la articulación de demandas implica el reconocimiento de un nosotros y que este se construye en la intersección de distintos discursos mediante el principio de equivalencia democrática, aquí nos proponemos, en primer lugar, indagar cómo actúa el lenguaje en la identificación de quienes combaten contra las diversas formas de dominación, así como también reconocer cuáles son los límites, desde dicha concepción, para la constitución de una identidad política colectiva amplia.

Y, en relación con ello, también resulta fértil para el debate la pregunta por el valor de asumir la ciudadanía, en términos pragmáticos, como espacio de articulación de una pluralidad de identidades políticas propia de los movimientos sociales, en el marco de la lucha que estos emprenden por la realización de los principios de igualdad y libertad.

Finalmente, a partir del análisis de estas cuestiones y estableciendo cierta tensión con la propuesta de Mouffe, desarrollamos un interrogante referido a las condiciones objetivas y subjetivas que determinarían, de cierto modo, cualquier posible convergencia de las disputas y las identidades de los actores que las emprenden. 


\section{Sobre identificaciones, articulaciones y equivalencias}

Siguiendo la propuesta de Mouffe (1999, 2001, 2011; Laclau y Mouffe, 2004), cualquier posibilidad de revolución democrática requiere una conexión de las distintas luchas que desafían las relaciones de dominación. Esta conexión se basa en la creación de nuevas posiciones subjetivas a partir de la articulación de las identidades de estas fuerzas en lucha.

Sin embargo, dicha convergencia no es espontánea sino que demanda el establecimiento de equivalencias que, mediante la creación de un nuevo sentido común, permitan articular las exigencias que estos grupos tienen en la lucha que han emprendido, y transformar, así, sus identidades, conformando un nosotros que los una y contenga.

Para Mouffe, este proceso se da mediante el principio de equivalencia democrática a través del cual los grupos (incluso aquellos que pudieran presentar demandas antitéticas) reconocen que tienen un interés común -la lucha por la extensión y la radicalización de la democracia- habilitando la construcción de una identidad colectiva, un nosotros, a partir de dicha identificación; esta idea, central en la propuesta de la autora, puede resumirse en la siguiente cita extraída de su texto "Feminismo, ciudadanía y política democrática radical": "El objetivo es construir un 'nosotros' como ciudadanos democráticos radicales, una identidad política colectiva articulada mediante el principio de equivalencia democrática" (Mouffe, 2001, p. 9).

Este principio al que Mouffe refiere actúa estableciendo una cadena de equivalencias entre las demandas de los distintos grupos, articulándolas en una nueva identidad común, algo diferente a una alianza por intereses particulares (Mouffe, 1999, p. 102; 2003). La noción de articulación, sin embargo, "... no elimina la pluralidad ni las diferencias y (...) respeta formas diversas de individualidad" (Mouffe, 1999, p. 141). En ese sentido, "es solo en la medida en que las diferencias democráticas se oponen a las fuerzas o discursos que niegan a todas ellas, que esas diferencias serán sustituibles entre sí" (Mouffe, 2001: 9).

Ahora bien, ¿̇cuál es el lugar del lenguaje en el proceso de identificación entre quienes combaten contra las diversas formas de dominación?

En la medida que las identidades de los sujetos se construyen de forma discursiva, cualquier forma de identificación será a través del lenguaje.

Laclau y Mouffe proponen un concepto que es clave para comprender el desarrollo de nuevos procesos de identificación: la subjetividad política refiere al modo en que "... los actores sociales actúan o toman decisiones novedosas" (Howarth, 1997, pp. 132-133). En este sentido, dado que las acciones de los sujetos se identifican con discursos de un modo precario, estos actúan de maneras diferentes en función de dicha contingencia, permitiendo una reconstrucción de sus identidades con base en articulaciones de discursos alternativos y la identificación con ellos.

En este punto del recorrido que hemos emprendido nos surge, a modo de pregunta casi inevitable, una primera advertencia: ¿cómo pensar aquellos procesos de articulación en los que podría derivar la conformación de una identidad amplia en la medida que su 
propia condición de precariedad define su labilidad en términos de permanencia? Retomaremos esta cuestión algunos párrafos más adelante.

Por ahora, conviene señalar que este carácter lábil y contingente de las identidades se relaciona con otro concepto clave en la teoría de Mouffe: las posiciones subjetivas refieren a las posiciones por la que puede optar el sujeto en diversos discursos constituyéndose de ese modo en un actor social con determinada identidad o identidades'.

Sin duda, esta concepción sobre la identidad echa por tierra cualquier definición esencialista en la medida que los grupos se constituyen sobre la base de posiciones de sujeto y no sobre identidades esenciales y permanentes.

Acordando con Mouffe, y descartada así la idea de cualquier esencialismo, la pregunta que aquí nos planteamos tiene que ver entonces con las posibilidades de emprender una revolución democrática radical sostenida por un nosotros cuya apertura, amplitud y profundidad garanticen su potencial transformador al tiempo que sean capaces de trascender su condición de contingente y precario.

\section{Sobre identidades, comunidades políticas y ciudadanía}

La idea de un nosotros construido a partir de la articulación de las identidades políticas de quienes luchan por la extensión y profundización de los principios democráticos lleva a la pregunta por el sujeto de dicha acción. En otras palabras, ¿̇cuál es esa forma de identificación que nos constituye como integrantes de una comunidad política? Mouffe encontrará la respuesta en el concepto de ciudadanía. En su libro El retorno de lo político. Comunidad, ciudadanía, pluralismo, democracia radical (1999) indicará, en relación con este concepto, que "El mismo implica no considerar la ciudadanía como estatus legal, sino como forma de identificación, un tipo de identidad política: algo a construir, no dado empíricamente" (1999, p. 96). Se trata de una identidad política común entre personas comprometidas en muy diversas empresas y con diferentes concepciones del bien, pero vinculadas las unas a las otras por su común identificación con una interpretación dada de un conjunto de valores ético-políticos (Mouffe, 2001, p. 8). Y, por tanto, habrá tantas formas de ciudadanía como interpretación de estas existan (Mouffe, 1999, p. 104).

En este sentido, en la medida que la ciudadanía está ligada al tipo de comunidad política en la que actúa, sus posibilidades transformadoras para la instauración de un orden democrático radical basado en los principios de libertad e igualdad estarán vinculadas a las probabilidades de creación de una cadena de equivalencias entre las distintas luchas orientadas a este fin; en palabras de la autora, "la visión de una democracia radical y plural que quiero proponer entiende la ciudadanía como una forma de identidad política que consiste en la identificación con los principios políticos de la democracia moderna pluralista, es decir, en la afirmación de la libertad y la igualdad para todos" (Mouffe, 2001, p. 8)

1 Ello, a su vez, se vincula con una concepción del agente social en tanto "articulación de un conjunto de posiciones de sujeto, correspondientes a la multiplicidad de las relaciones sociales en que se inscribe." (Mouffe, 2001, p. 7) 
Sin duda, este concepto de ciudadanía permite comprender, en términos pragmáticos, las distintas luchas que movimientos sociales llevan a cabo por subvertir el orden de dominación. En tanto "principio de articulación que afecta a las diferentes posiciones subjetivas del agente social (...), aunque reconociendo una pluralidad de lealtades específicas y el respeto a la libertad individual" (Mouffe, 1999, p.101), la ciudadanía hace posible el agrupamiento de aquellos actores que luchan por la extensión y profundización de los principios democráticos, configurándose en una identidad política común. Sin embargo, nos interesa señalar aquí que en esta propuesta queda latente la pregunta por las potencialidades y los límites vinculados a las posibilidades de establecer efectivas equivalencias entre las distintas interpretaciones que los actores otorgan a dichos principios. Las posibilidades de construcción y reconstrucción de las identidades con base en sus identificaciones precarias y contingentes con los discursos permiten asumir la ciudadanía de un modo activo en tanto es capaz de modificarse según articulaciones discursivas alternativas. Pero es este mismo carácter activo -inherente a ella, por cierto- el que nos lleva nuevamente a la pregunta, central en este artículo, por las posibilidades de permanencia de un nosotros constituido como identidad política, en orden de viabilizar cualquier proceso de expansión y profundización de una democracia radical.

\section{La revolución ¿un sueño eterno?}

Y es, justamente, en vínculo con esa pregunta, la cuestión que aquí proponemos repensar en la propuesta de Mouffe: la misma tiene que ver con las condiciones subjetivas y objetivas bajo las cuales actúan los distintos grupos sociales que luchan por la expansión del orden democrático, habilitando la configuración de esa identidad política común.

Desde el campo de estudios de la comunicación y la política, María Cristina Mata (2006) propone dichas nociones como cuestiones centrales para el análisis de las prácticas de reconocimiento, ejercicio y búsqueda de ampliación del derecho a la comunicación, esto es, las prácticas de ciudadanía comunicativa. Las condiciones objetivas, afirma la autora, refieren a las condiciones económicas y políticas de cada coyuntura particular, así como a las condiciones culturales que la posibilitan o limitan. Al mismo tiempo, existen múltiples condiciones subjetivas, que involucran los modos de imaginarse como sujetos de derecho, propios de diferentes individuos y colectivos sociales, sus maneras de vincularse con quienes detentan la legalidad y legitimidad social para expresarse y producir normas al respecto, las representaciones hegemónicas y contrahegemónicas acerca de lo que significa comunicarse y comunicar hoy en sociedades mediatizadas, las expectativas depositadas en las instituciones, las propias fuerzas expresivas.

A partir de estas dos categorías desarrolladas por Mata, y asumiendo que toda práctica social (y con ello, toda práctica política) está condicionada subjetiva y objetiva mente, nos interesa en este trabajo reflexionar sobre el modo en que se ponen en juego dichas categorías en el desarrollo teórico de Chantal Mouffe, al momento de pensar en las posibilidades y modalidades que asume el proceso de subjetivación política en el cual se configuraría una nueva identidad, en lucha contra las distintas formas de opresión. 
La fortaleza de las categorías de condiciones subjetivas y objetivas está en que permiten incorporar al análisis de las prácticas de los movimientos sociales, de manera complementaria con la noción de posición subjetiva ${ }^{2}$ desarrollada por Mouffe, aquellos factores que posibilitarían o dificultarían -en diferentes grados y maneras- la configuración de una identidad común amplia para la lucha en favor de los principios democráticos.

En este marco, la idea de Mouffe respecto de las identificaciones de los agentes es susceptible de ser comprendida como una condición subjetiva para el accionar. Plantea la autora que las posibilidades de acción de los sujetos están dadas por el carácter precario y contingente de las identificaciones, y en el mismo sentido, que su posibilidad de articulación se vincula con el reconocimiento del carácter particular y limitado de sus reivindicaciones. Así lo afirma cuando analiza los movimientos feministas: "Considero que el esencialismo conduce a una visión de la identidad que no concuerda con una concepción de democracia plural y radical y que no nos permite construir la nueva visión de la ciudadanía que hace falta para aplicar tal política" (Mouffe, 1999, p.109). En este sentido, el falso dilema de la igualdad frente a la diferencia se derrumba desde el momento en que se asume que no hay, por ejemplo, una entidad homogénea «mujer» enfrentada con otra entidad homogénea «varón», sino una multiplicidad de relaciones sociales en las cuales la diferencia sexual -o cualquier otra diferencia- está construida siempre de muy diversos modos, y donde las luchas en contra de la subordinación tienen que plantearse de formas específicas y diferenciales (Mouffe, 1999). De este modo, lo que Mouffe plantea es que en el dominio de lo político, y por lo que toca a la ciudadanía, la diferencia sexual, étnica, etc. no debe ser una distinción pertinente, y propone una concepción diferente de qué es ser un ciudadano y cómo actuar en tanto miembro de una comunidad política democrática.

Por otro lado, la relativa contingencia, variabilidad e historicidad que tanto Mouffe ${ }^{3}$ como Laclau le asignan a los vínculos entre los diversos agentes puede ser comprendida en términos de condición objetiva para la articulación.

Sin embargo, lo que aquí pretendemos poner en tensión en esa propuesta teórica es que el análisis de las prácticas políticas de movimientos sociales desde una perspectiva diacrónica permite reconocer una cuestión no mencionada en la propuesta de Mouffe: el hecho de que en un determinado momento histórico diferentes grupos pueden luchar contra una determinada configuración de poder que genera múltiples formas de opresión no puede ocultar que ese poder, al igual que las identidades, tiene carácter histórico; y esto es significativo en la medida que sus posibles institucionalizaciones han vinculado a los diversos grupos en cuestión de maneras diferentes a lo largo de la historia.

2 Con la categoría de posición subjetiva Mouffe y Laclau refieren a la "... posición por la que opta el sujeto en diversos discursos. Esto significa que los individuos pueden tener varias posiciones subjetivas. Un determinado agente empírico puede considerarse a sí mismo 'negro', 'de la clase obrera', 'cristiano', 'mujer', ecologista', etc. [Así,] la noción de posición subjetiva se ocupa de las múltiples formas que tienen los agentes de constituirse a sí mismos en actores sociales..." (Howarth, 1997, p.32).

3 En ese sentido, Mouffe (1999) reconoce, siguiendo a Oakeshott, la incidencia de las tradiciones de conducta de los sujetos en sus acciones políticas. 
Ante esta situación surge la pregunta por las posibilidades y modalidades de articulación entre grupos que hoy comparten una lucha particular en el marco de la disputa por la instauración de los principios democráticos, pero que actualmente o en otros momentos han ocupado posiciones relativas desiguales en el marco de relaciones de opresión. Cuestiones como estas no deberían de omitirse en el análisis de las prácticas políticas que movimientos sociales particulares llevan a cabo en un momento histórico determinado.

En el mismo sentido, desde una perspectiva sincrónica, es importante considerar que esta articulación, este proceso de subjetivación política, involucraría actores que no solo parten de posiciones subjetivas diferentes sino que, además, de modo probable, ocupan posiciones desiguales en el marco de relaciones de poder, pudiendo estar vinculados a las distintas trayectorias particulares, modalidades de organización, marcos normativos, coyunturas político-estatales, etcétera.

Esto puede observarse en las relaciones que se establecen entre distintos movimientos sociales hoy en América Latina. En el caso de los movimientos campesinos y los movimientos indígenas ${ }^{4}$, por abordar solo un ejemplo, la reconstrucción de las trayectorias y modos en que se han ido transformando sus demandas, sus modos de identificación y los vínculos entre ambos sectores en el marco de transformaciones políticas y económicas más amplias, permite comprender las dificultades que en algunos casos han surgido en el proceso de articulación de sus demandas: dificultades relacionadas con el hecho de que en distintos momentos históricos estos grupos han ocupado posiciones relativas desiguales en el marco de relaciones de opresión más generales, asumiendo, en consecuencia, posiciones subjetivas diferentes.

Históricamente, los grupos indígenas y sus demandas particulares fueron invisibilizados tanto por los Estados nacionales, como también en el marco de algunos procesos políticos revolucionarios protagonizados por ciertos sectores de la izquierda latinoamericana 5 . Enmarcadas en estos procesos políticos, las luchas indígenas se subsumieron a las lógicas de la lucha de clase y fueron asumidas como luchas campesinas. Para fines de la década de 1980, sin embargo, esto comenzó a ser cuestionado por los movimientos

4 Abordamos aquí el caso de estos movimientos en tanto constituyen las temáticas de las investigaciones de doctorado que realizamos las autoras de este artículo. Una de ellas se centra en el Movimiento Campesino de Córdoba y la otra en distintas organizaciones indígenas del actual territorio argentino. En ambos casos, las investigaciones implican el estudio de la historia de esas organizaciones, las trayectorias de sus prácticas políticas, así como las modalidades de vínculo con otros movimientos y organizaciones.

5 A fines de los años 50 algunos gobiernos de América Latina -como el caso de Bolivia (1953), Chile (1962), Ecuador (1964) o Perú (1969)- impulsaron, con diferencias en cada caso, reformas agrarias que contribuyeron a cambiar en alguna medida las condiciones de vida de los campesinos, y que en muchos casos fueron la plataforma para el desarrollo de organizaciones campesinas e indígenas (Bello, 2004: 63). Sin embargo, muchas de estas organizaciones que surgían en los años 50 estuvieron vinculadas desde su origen a intelectuales y organizaciones de la izquierda latinoamericana, y por lo general desde esos sectores -salvo el caso de algunos pensadores como José Mariátegui en Perú o Guzmán Böckler en Guatemala- existieron ciertas reticencias para aceptar el hecho de una movilización política indígena como una entidad propia, con lo cual la tendencia fue integrar las luchas indígenas y las reflexiones sobre las mismas en la lógica de lucha campesina. 
indígenas. A partir de entonces, la configuración de nuevos regímenes de visibilidad de la cuestión étnica (Bengoa, 2009) y la progresiva legitimación de los indígenas en tanto sujetos de derecho internacional ${ }^{6}$ les han otorgado determinadas posibilidades de visibilización de sus luchas, así como también de reivindicación de derechos particulares vinculados a la indigenidad (tal como el derecho ancestral sobre las tierras en que viven, la autonomía política en los propios territorios, el derecho a la educación bilingüe, por citar algunos ejemplos), que resultan relativamente más significativas que las obtenidas por otros movimientos, como por ejemplo, los campesinos. Este proceso ha dado lugar, en algunos casos, a dificultades para la articulación de las luchas de estos dos sectores, e incluso, a veces, a posiciones enfrentadas respecto de los términos y modos en que se plantean y defienden las reivindicaciones referidas a cuestiones que les resultan centrales, como lo es el derecho al territorio.

En suma, lo que aquí se pretende enfatizar mediante la ejemplificación del caso de movimientos indígenas y campesinos de la región es que en el análisis de las prácticas políticas de los distintos movimientos sociales se torna central considerar cuál es la incidencia que tienen las condiciones objetivas y subjetivas (históricamente configuradas) que determinan dichas prácticas, y esto tanto en el proceso de articulación y constitución de una identidad amplia que los comprenda, como en el ejercicio de la práctica política revolucionaria.

En relación con ello, Mouffe plantea que la configuración de una frontera que vincule a estos sujetos dentro de un nosotros permite dejar de lado otras cuestiones que contraponen a los actores. Desde esta perspectiva, los discursos adquieren su identidad mediante el trazado de fronteras políticas y la construcción de relaciones agonísticas entre ese nosotros y otros que se configuran en tanto adversarios ${ }^{7}$ en la comunidad política. Ello implica, a su vez, el reconocimiento, en el marco de la democracia, de que el enfrentamiento agonal, lejos de representar un peligro, es en realidad su condición de existencia puesto que habilita la emergencia de apuestas democráticas en torno a las cuales puedan cristalizar las identificaciones colectivas. Ello se materializa, según esta propuesta, en la posibilidad de que se instauren las condiciones de un pluralismo agonístico que permita reales confron-

6 De todos modos, pese a estas transformaciones puede observarse que en general la distribución de la población indígena coincide con las áreas de los países donde se localizan los mayores índices de necesidades básicas insatisfechas (Mombello, 2002: 5), lo cual indica que la brecha que separa la letra de la ley de las políticas públicas es aún muy grande. Ello se vincula también con el hecho de que este proceso de reconocimiento de las poblaciones indígenas y sus derechos ha sido concomitante con "las reformas del Estado en el Cono Sur durante la década de los 90’s, orientadas a la desregulación y retirada de la injerencia estatal en la provisión de servicios y coberturas sociales básicas. Así, estas políticas han operado en un sentido inverso al reconocimiento de los derechos indígenas, pues contribuyeron a la exclusión y pauperización de las poblaciones más vulnerables y entre ellas los pueblos originarios" (Trinchero, 2009).

7 En este punto, Mouffe distingue entre el antagonismo, como relación con el enemigo y el agonismo, en tanto relación con el adversario. Eso significa que en el interior del nosotros que constituye la comunidad política, no se verá en el oponente un enemigo a abatir, sino un adversario de legítima existencia, cuyas ideas se combatirán pero no se cuestionará su derecho a defenderlas. Sin embargo, la categoría de enemigo no desaparece, ya que sigue siendo pertinente en relación con quienes, al cuestionar las bases mismas del orden democrático, no pueden entrar en el círculo de los iguales (Mouffe, 1999, p. 16). 
taciones en el seno de un espacio común, con el fin de que puedan realizarse verdaderas opciones democráticas (Mouffe, 1999, p.18).

Sin embargo, como dijimos, esta respuesta no debería conducir a dejar de lado, en el análisis de los procesos políticos, el abordaje del carácter histórico de los vínculos entre las distintas luchas (muchas veces tejidos en relaciones de desigualdad), y de la incidencia que esto tiene en las posibilidades de confluencia en un momento determinado.

Al mismo tiempo, si se considera que "... la experiencia del antagonismo muestra de manera esencial la contingencia de la identidad" (Howarth, 1997, p.131), se deriva nuevamente en la pregunta que aquí nos planteamos por la permanencia y la trascendencia, dado que las asociaciones contingentes entre quienes luchan por un determinado objetivo político podrían volverse meras asociaciones temporales, lo cual no solo amenaza la profundidad de los movimientos y sus reivindicaciones, sino también la permanencia de sus resultados (Martínez Labrín, 2006, p.62).

\section{Palabras finales}

La posibilidad de construcción de una nueva hegemonía de los valores democráticos a partir de la articulación de las diferentes luchas contra los múltiples modos de opresión se produciría en, por y desde el lenguaje ${ }^{8}$. Se trata de la configuración de nuevas identidades, de un nuevo sujeto, el ciudadano democrático. Esta forma de identificación sólo es concebible en el contexto de un nuevo tipo de articulación entre lo universal y lo particular, de acuerdo con la modalidad de un universalismo que integre las diversidades.

Y en ese punto, Mouffe afirma que para ello debe entrar en escena la multiplicación de las posiciones de sujeto democráticas según dispositivos que permitan a las diferentes posiciones enfrentarse en el seno de lo que reconocen como constitutivo de su espacio político común?.

Sin embargo, la pregunta que aquí nos planteamos -y cuya respuesta debe buscarse en el marco del análisis de las luchas políticas históricamente situadas- es sobre el modo en que podría crearse esa confluencia revolucionaria de las distintas luchas cuando las relaciones entre ellas no están condicionadas solo por la diversidad sino también por desigualdades. Es posible que la respuesta refiera al carácter contingente de la articulación. Y allí, entonces, emerge la interrogación por la profundidad de los movimientos y sus reivindicaciones y por la permanencia de sus acciones y potenciales resultados. Nuevamente, se trata de reflexiones a construir a partir de la consideración de las luchas políticas históricamente situadas, de aquellas que derivan en antagonismos irreconciliables y aquellas que se consolidan, perduran y reconfiguran cotidianamente.

8 Ello, por supuesto, sin caer en las posturas que desde la filosofía política pugnan por la posibilidad de un consenso racional, ni en la propuesta habermasiana sobre la situación ideal de comunicación.

9 Por el contrario, "... cuando el espacio público democrático se debilita, se ve cómo se multiplican los enfrentamientos en términos de identidades esencialistas o de valores morales no negociables." (Mouffe, 1999, p. 18). 
Sin duda, la actual experiencia popular caribeña y latinoamericana, hecha de desigualdades, diferencias, antagonismos y contradicciones, pero también de luchas genuinas territorialmente situadas, amalgamadas con convicciones, voluntades y anhelos comunes, está brindando al pensamiento en las ciencias sociales una gran oportunidad para esa reflexión.

\section{Referencias bibliográficas}

Bello, Álvaro (2004) Etnicidad y ciudadanía en América Latina. La acción colectiva de los pueblos indígenas. Chile: CEPAL y Sociedad Alemana de Cooperación Técnica (GTZ).

Bengoa, José (2009) "¿Una segunda etapa de la Emergencia Indígena en América Latina?”, en Cuadernos de Antropología Social n. ${ }^{\circ}$ 29. Buenos Aires: Facultad de Filosofía y Letras-UBA. pp. 7-22.

Howarth, David (1997) La teoría del discurso. En Marsh, David y Stoker, Ferry (eds.), Teoría y métodos de la ciencia política. Madrid: Alianza Editorial S. A. pp. 127-142.

Laclau, Ernesto y Mouffe, Chantal (2004) Hegemonía y estrategia socialista. Hacia una radicalización de la democracia. Buenos Aires: Fondo de Cultura Económica.

Martínez Labriz, Soledad (2006) Política democrática radical y feminismo. En Athenea Digital núm. 009. pp. 58-64.

Mata, María Cristina (2006) Comunicación y ciudadanía. Problemas teórico-políticos de su articulación. Fronteiras - estudos midiáticos, VIII (1). pp. 5-15.

Mombello, Laura (2002) Evolución de la política indigenista en Argentina en la década de los noventa. CLAPSO, Universidad de Texas. http://lanic.utexas.edu/project/laoap/claspo/dt/0004.pdf

Mouffe, Chantal (1999) El retorno de lo político. Comunidad, ciudadanía, pluralismo, democracia radical. Barcelona: Paidós.

Mouffe, Chanta (2001) "Feminismo, ciudadanía y política democrática radical". En Lamas, Marta (comp.) (2001) Ciudadanía y feminismo. Fondo de Desarrollo de las Naciones Unidas para la Mujer (VNIFEM) y del Instituto Federal Electoral (IFE). México. pp. 2-13.

Mouffe, Chantal (2011) La política y lo político. En: En torno a lo político. Buenos Aires: Fondo de Cultura Económica. pp. 15-40.

Trinchero, Héctor H. (2009) "Pueblos originarios y políticas de reconocimiento en Argentina". En: Papeles de Trabajo n. ${ }^{\circ}$ 18. Rosario: Centro de Estudios Interdisciplinarios en Etnolingüística y Antropología Socio-Cultural. pp. 1-17. 
